\title{
Cholecystokinin Expression in the Development of Postinfarction Heart Failure
}

\author{
Xiaoying Dong Can Wang Jingqi Zhang Siyue Wang Hongjian Li Yang Kang \\ Shuangquan Tian Lu Fu
}

Laboratory of Cardiovascular Medicine, Department of Internal Medicine, First Affiliated Hospital, Harbin Medical University, Heilongjiang, China

\section{Key Words}

Cholecystokinin - Myocardial infarction - Heart failure - Brain natriuretic peptide • Gastrointestinal hormone

\begin{abstract}
Background/Aims: Cholecystokinin (CCK) is expressed in cardiomyocytes and may also play an important role in cardiovascular regulation. Clinical studies have shown that plasma CCK levels are an independent marker of cardiovascular mortality in cardiac disease. However, whether the development of postinfarction heart failure is associated with changes in CCK expression is unknown. Methods: To investigate CCK expression patterns and the association between CCK expression and heart functional parameters, we randomized male SpragueDawley rats into myocardial infarction (MI) or sham operation (SO) groups. CCK expression levels were assessed by western blotting, immunohistochemistry, real-time polymerase chain reaction (PCR), and enzyme-linked immunosorbent assay (ELISA) at different time points $(2,4$ or 6 weeks) after surgery. Brain natriuretic peptide (BNP) concentrations were determined using Western blotting and ELISA, myocardial morphology was assessed by microscopy. Results: Plasma CCK and BNP levels were significantly increased in all the MI groups compared with the corresponding SO groups. However, the degree to which myocardial CCK mRNA and protein expression levels were increased the MI groups compared with the SO groups was greater in the 4- and 6-week groups than in the 2-week group. Furthermore, plasma CCK levels were positively correlated with BNP concentrations and left ventricular end-systolic diameter (LVDs) and significantly negatively correlated with the ejection fraction (EF) and shortening fraction (SF) in model animals. Conclusions: Heart failure progression after infarction is associated with upregulated CCK levels; thus, CCK may be useful as a novel marker of heart failure.
\end{abstract}




\section{Cellular Physiology Cell Physiol Biochem 2017;43:2479-2488

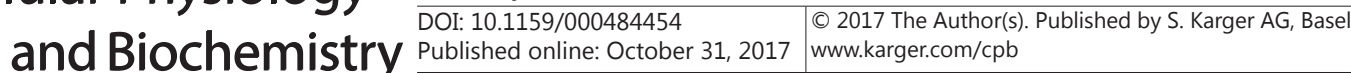

Dong et al.: Expression of Cholecystokinin During Heart Faiure Development

\section{Introduction}

Chronic heart failure (CHF) is not merely a complex syndrome. Rather, CHF is a complicated pathophysiologic process whose development has been attributed to several mechanisms. Hormonal imbalances may be important contributors to worse prognoses in patients with heart failure [1,2]. Researchers have become increasingly interested in using appetite-regulated hormones, such as orexigenic peptides (ghrelin)[3-5] and anorexigenic peptides (leptin)[6], as biomarkers of cardiac and systemic abnormalities and as potential drugs in the treatment of heart failure, especially in cardiac cachexia [4, 7].

Cholecystokinin (CCK) is another appetite-regulated hormone that is widely distributed throughout various organs and tissues [8-10] and exists in various molecular forms (CCK-58, CCK-33, CCK-22, CCK-8, and CCK-4)[11]. However, the variation in bioactive CCKs is small in among species. In addition to mediating gallbladder contraction, pancreatic secretion, memory, and satiation $[12,13]$, CCK has also been shown to exert protective effects in the lung [14], kidney [15], liver [16] and heart through its receptors (CCK-AR/BR) in recent studies [17]. The multifunctionality of CCK suggests that it may have potential as a target in the evaluation and treatment of various diseases [18-20]. A recent study demonstrated that CCK was expressed at high concentrations in cardiac myocytes [21] despite being synthesized predominantly in the gastrointestinal tract and central nervous system [10]. These findings indicate that CCK has a variety of effects as a novel cardiovascular hormone. Previous studies have shown that CCK regulates blood pressure [22-24] and increases cardiac contractility [25]. Moreover, clinical studies have reported that plasma CCK levels are linked to cardiovascular mortality; thus, CCK concentrations may be useful as valuable biomarkers in elderly females with symptoms of cardiac disease, as shown in a previous study [26]. These findings suggest that CCK may play an important role in the development of cardiovascular diseases. However, to our knowledge, whether the development of heart failure is associated with changes in CCKexpression is unknown. In addition, the relationships between plasma CCK expression and heart functional parameters and brain natriuretic peptide (BNP) concentrations remain unknown. Thus, the present study was conducted to analyse CCK expression patterns in the animal model of left anterior descending coronary artery (LAD) ligation, and to determine the correlation between CCK expression and heart functional parameters. This work demonstrated that CCK expression is correlated with myocardial injury in postinfarction heart failure.

\section{Materials and Methods}

\section{Animals}

Male Sprague-Dawley rats ( $\mathrm{n}=60$, weighing 200-220 g, 6-7 weeks of age) were obtained from the Laboratory Animal Center of the First Affiliated Hospital of Harbin Medical University. Four rats were housed per cage under standard conditions (temperature $22-24^{\circ} \mathrm{C}$ humidity $50 \pm 5 \%$, light from 8 a.m. to 8 p.m.) and allowed access to food and water ad libitum. All investigations conformed to the Guide for the Care and Use of Laboratory Animals of the National Research Council, and the protocol for this study was reviewed and approved by the ethics committee of our hospital.

Rat model of heart failure

After a 7-day acclimation period, the animals were randomly subjected to LAD ligation (MI) or sham operation (SO). The models were established as previously described [27], with minor modifications. Briefly, the rats were anaesthetized with $10 \%$ chloral hydrate $(3 \mathrm{ml} / \mathrm{kg}$, intraperitoneal injection), endotracheally intubated with a 14-gauge angiocath, and then mechanically ventilated with a ventilator. LAD ligation was performed through the fourth intercostal space, and the proximal LAD was ligated with 6-0 sutures. Successful LAD ligation was signified by the presence of changes consistent with ST-segment elevation on electrocardiography and colour changes in the ischaemic area. SO animals underwent the same procedure but did not undergo ligation. All surgeries were performed using sterile techniques. Three rats died during 


\section{Cellular Physiology Cell Physiol Biochem 2017;43:2479-2488

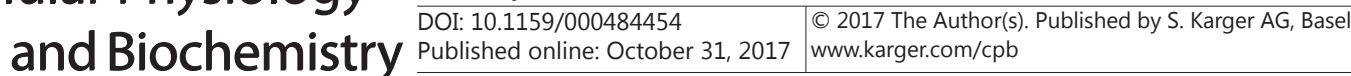

Dong et al.: Expression of Cholecystokinin During Heart Faiure Development

surgery. Post-operatively, the surviving MI and SO rats were randomly assigned to the following groups: (A) a 2-week group (n=9/9, MI/SO), (B) a 4-week group (n=9/10) and (C) a 6-week group (n=10/10).

\section{Echocardiography}

Echocardiography was performed under $10 \%$ chloral hydrate anaesthesia that was injected intraperitoneally. The rats were placed in the appropriate position after their fur was shaved and their skin was cleaned, and then two-dimensional M-mode echocardiography was performed to assess cardiac function and to measure several structural parametersin both the parasternal long- and short-axis views using an ultrasound machine (SONOS 7500, Philips) fitted with a 12-MHz transducer. Left ventricular end-systolic diameter (LVDs) and left ventricular end-diastolic diameter (LVDd) were measured in three consecutive cardiac cycles, after which the average value of each parameter was recorded. Left ventricular fractional shortening (LVFS) and the left ventricular ejection fraction (LVEF) were calculated using the following formulas: $\operatorname{LVFS}(\%)=(\mathrm{LVDd}-\mathrm{LVDs}) / \mathrm{LVDd} \times 100 \%$ and $\operatorname{LVEF}(\%)=\left(\mathrm{LVDd}^{3}-\mathrm{LVDs}^{3}\right) / \mathrm{LVDd}^{3} \times 100 \%[28]$.

\section{Sample collection}

After the above echocardiographic parameters were measured, blood was collected from the abdominal aorta and centrifuged to obtain plasma. The rats were subsequently euthanized, and their hearts were rapidly removed and irrigated with cold saline solution. The weight of each heart was measured, and the ratio of heart weight to body weight (HW/BW) was calculated. A portion of the myocardium was fixed in $4 \%$ paraformaldehyde for further histological analysis, while duodenal samples, which ultimately served as a positive control in subsequent protein and gene expression analyses, and the remaining myocardial samples were quickly frozen in liquid nitrogen and stored at $-80^{\circ} \mathrm{C}$.

\section{Morphological changes in the myocardium}

Transverse sections of the cardiac apex were cut into 5 - $\mu$ m-thick sections and stained with haematoxylin and eosin (H\&E) to analyse LAD ligation-induced changes in myocardial morphology and Masson's trichrome to evaluate the severity of myocardial fibrosis. Three slides each were randomly selected from three rats in each group for morphological analysis.

\section{Real-time PCR}

CCK mRNA expression levels in the noninfarcted area of the left ventricle were detected by semiquantitative reserve transcription polymerase chain reaction (RT-PCR). Total RNA was extracted with an RNA Extraction Kit (Takara Bio, Otsu, Japan), and cDNA was synthesized using a PrimeScript ${ }^{\mathrm{TM}}$ RT Reagent Kit with gDNA Eraser (Takara Bio, Otsu, Japan), according to the manufacturer instructions. CCK amplification was performed with SYBR Green on an ABI 7500 Real-Time PCR System (Applied Biosystems, Foster, CA, USA). The following primer sequences were used for this experiment: CCK: forward: 5'-CCC GAT ACA TCC AGC AGG TC-3' and reverse: 5'-AAA TCC ATC CAG CCC ATG TAG TC-3'; and GAPDH: forward: 5'-GGC ACA GTC AAG GCT GAG AAT G-3'and reverse: 5'-ATG GTG GTG AAG ACG CCA GTA-3'. GAPDH was used as an internal standard. The relative expression levels of CCK were determined using the $2^{-\triangle \triangle \mathrm{Ct}}$ method.

\section{Western blotting}

To measure endogenous CCK and BNP expression levels in cardiomyocytes, we extracted proteins from noninfarcted left ventricular tissue samples, diluted them with $5 \times$ buffer and measured protein concentrations using a protein assay kit (BCA, Beyotime Institute of Biotechnology, Haimen, China). The proteins were then subjected to heat denaturation before being separated on $12 \%$ SDS-polyacrylamide gels and then transferred to PVDF membranes, which were blocked for 1 hour at room temperature before being incubated with primary antibodies against CCK(1:800; Sigma-Aldrich, Saint Louis, USA), BNP(1:4, 000; WanLeiBiotechnology, ShengYang, China), and GAPDH(1:4, 000; ZhongShan, Beijing, China) overnight at $4^{\circ} \mathrm{C}$. The membranes were then incubated with the appropriate horseradish peroxidase-conjugated secondary antibodies (anti-mouse IgG 1:2, 000 or anti-rabbit IgG 1:2, 000; ZhongShan, Beijing, China) for 1 hour at room temperature. All images were captured and analysed using Image Lab software (Bio-Rad Universal Hood II, USA). The expression levels of the above proteins were normalized to those of GAPDH. All experiments were performed three times.

\section{KARGER}




\section{Cellular Physiology Cell Physiol Biochem 2017;43:2479-2488 \begin{tabular}{l|l|l} 
and Biochemistry Published onlıne: October 31, 2017 & $\begin{array}{l}\text { (c) } 2017 \text { The Author(s). Published by S. Karger AG, Basel } \\
\text { www.karger.com/cpb }\end{array}$
\end{tabular} \\ Dong et al.: Expression of Cholecystokinin During Heart Faiure Development}

Enzyme immunoassay (EIA) for CCK and BNP expression measurements

Plasma CCK and BNP levels were measured with commercially available enzyme-linked immunoassay kits (RayBiotech, Inc., Norcross, GA), according to the manufacturer's protocol. The lower limits of detection for CCK and BNP were $0.2 \mathrm{pg} / \mathrm{ml}$ and $4.7 \mathrm{pg} / \mathrm{ml}$, respectively. The coefficient of variation was $<10 \%$.

Immunohistochemistry

For the CCK immunostaining experiments, myocardial tissue samples were fixed with $4 \%$ paraformaldehyde, embedded in paraffin and then sectioned at a thickness of $4 \mu \mathrm{m}$. The sections were subsequently de-paraffinized, rehydrated and then incubated with a rabbit anti-rat antibody against CCK (1:100, Cloud-Clone Corp, WuHan, China) overnight at $4^{\circ} \mathrm{C}$. The sections were then counterstained with haematoxylin and observed under a fluorescence microscope (OLYMPUS DP73, Japan).

\section{Statistical analysis}

The results are presented as the mean \pm standard deviation (SD). Comparisons between two groups were performed using independent samples t-tests (two-sided). ANOVA of a factorial design was used to assess the differences among groups. If the interactions among the groups were not significant, the ANOVA was followed by a least significant difference (LSD) test; however, if the interactions among the groups were significant, the ANOVA was followed by a simple effect analysis. Pearson's correlation coefficient was used to study the associations between CCK expression and heart functional parameters. Statistical analysis was performed using SPSS 20.0 software (SPSS Inc, Chicago, IL, USA). P $<0.05$ was considered statistically significant. To reduce subjective bias when assessing the results, the data collectors, outcomes adjudicators, and data analysts were blinded by labeling the groups with nonidentifying terms (A or B) until the entire analysis was completed.

\section{Results}

\section{Experimental model}

Forty-two surviving animals ( 8 animals in the 2-week SO group, 9 animals in the 4-week SO group, 8 animals in the 6-week SO group, 5 animals in the 2-week MI group, 6 animals in the 4-week MI group, and 6animals in the 6-week MI group) completed this study.

\section{Body weights and cardiac functional and structural parameters}

The cardiac morphological and functional characteristics of the rats were measured via echocardiography (Table 1). Body weight was significantly lower $(\mathrm{P}<0.001)$, and IVSTd was thinner $(\mathrm{P}<0.01)$ in the 6-week MI group than in the corresponding S0 group. LA $(\mathrm{P}<0.05)$, LVDd $(\mathrm{P}<0.001)$, and the HB/BW ratio $(\mathrm{P}<0.001)$ were significantly higher in the 4-week MI group than in the 4-week SO group, and LVDs was significantly greater in all the MI groups than in the corresponding SO groups. However, LVFS and LVEF were significantly decreased at all time points in the MI group compared with the SO group. No significant differences in LVPWTd were observed between two groups at any of the above time points.

\section{Myocardial degeneration and fibrosis}

H\&E and Masson's trichrome staining showed (Fig. 1) that significant inflammatory infiltrates, cardiomyocyte derangement, and collagen fibre deposition were present in the MI groups at all time points. Moreover, leukocytic infiltration was more pronounced in the2week MI group than in the 4- and 6-week MI groups. More severe cardiomyocyte hypertrophy and degeneration and myocardial interstitial fibrosis were observed in the 6-week MI group than in the other groups.

\section{Measurement of plasma CCK and BNP levels}

Plasma CCK and BNP levels were measured by ELISA (Fig. 2), which showed that plasma BNP levels were significantly higher in the MI groups than in the corresponding SO groups $(\mathrm{P}<0.001)$. Plasma CCK concentrations were also increased in the MI groups compared with 


\section{Cellular Physiology Cell Physiol Biochem 2017;43:2479-2488 \begin{tabular}{l|l|l} 
DOI: 10.1159/000484454 & Ond Biochemistry Published online: October 31, 2017 & $\begin{array}{l}\text { 2 2017 The Author(s). Published by S. Karger AG, Basel } \\
\text { www.karger.com/cpb }\end{array}$
\end{tabular}

Table 1. Body weights and cardiac functional and structural parameters in the rat models at the end of the study. BW: body weight; HW: heart weight; LA diameter: left atrial diameter; IVSTd: end-diastolic interventricular septal thickness; LVDd: left ventricular end-diastolic diameter; LVDs: left ventricular end-systolic diameter; LVPWTd: left ventricular end-diastolic posterior wall thickness; LVEF: left ventricular ejection fraction; LVFS: left ventricular short-axis fractional shortening; MI: myocardial infarction group. Data are expressed as the mean \pm standard deviation (SD). ${ }^{*} \mathrm{P}<0.05$, ${ }^{* *} \mathrm{P}<0.01,{ }^{* * *} \mathrm{P}<0.001$, compared with the timematched SO groups

\begin{tabular}{|c|c|c|c|c|c|c|}
\hline \multirow{2}{*}{ Parameters } & \multicolumn{2}{|c|}{2 Weeks } & \multicolumn{2}{|c|}{4 Weeks } & \multicolumn{2}{|c|}{6 Weeks } \\
\hline & Sham & MI & Sham & $\mathrm{MI}$ & Sham & MI \\
\hline $\mathrm{BW}(\mathrm{g})$ & $288.20 \pm 20.33$ & $297.60 \pm 23.93$ & $317.20 \pm 23.49$ & $293.40 \pm 18.08$ & $385 \pm 14.58$ & $325.00 \pm 16.25^{* * *}$ \\
\hline $\mathrm{HW} / \mathrm{BW}(\mathrm{mg} / \mathrm{g})$ & $3.19 \pm 0.20$ & $3.38 \pm 0.15$ & $3.06 \pm 0.18$ & $3.86 \pm 0.22 * * *$ & $2.61 \pm 0.09$ & $3.41 \pm 0.12 * * *$ \\
\hline $\mathrm{LA}(\mathrm{mm})$ & $2.91 \pm 0.20$ & $3.04 \pm 0.27$ & $2.99 \pm 0.19$ & $3.60 \pm 0.43^{*}$ & $3.04 \pm 0.18$ & $3.12 \pm 0.33$ \\
\hline $\operatorname{IVSTd}(\mathrm{mm})$ & $0.85 \pm 0.11$ & $0.88 \pm 0.05$ & $0.83 \pm 0.03$ & $0.72 \pm 0.10$ & $0.86 \pm 0.02$ & $0.75 \pm 0.05^{* *}$ \\
\hline $\operatorname{LVDd}(\mathrm{mm})$ & $6.18 \pm 1.29$ & $7.91 \pm 1.16$ & $5.42 \pm 0.45$ & $8.15 \pm 0.73 * * *$ & $7.15 \pm 0.69$ & $8.14 \pm 1.08$ \\
\hline LVDs(mm) & $3.61 \pm 0.91$ & $5.94 \pm 0.90 * *$ & $2.95 \pm 0.19$ & $7.30 \pm 1.22 * * *$ & $4.44 \pm 0.59$ & $6.75 \pm 1.03 * *$ \\
\hline $\operatorname{LVPWTd}(\mathrm{mm})$ & $0.93 \pm 0.07$ & $0.94 \pm 0.09$ & $0.94 \pm 0.05$ & $0.94 \pm 0.40$ & $1.00 \pm 0.03$ & $0.97 \pm 0.04$ \\
\hline $\operatorname{LVEF}(\%)$ & $77.98 \pm 5.30$ & $49.96 \pm 13.80 * *$ & $81.82 \pm 4.00$ & $28.46 \pm 9.57 * * *$ & $72.92 \pm 5.52$ & $40.98 \pm 4.20 * * *$ \\
\hline LVFS(\%) & $40.92 \pm 6.10$ & $24.72 \pm 6.36 * *$ & $45.36 \pm 4.41$ & $13.46 \pm 5.60 * * *$ & $37.990 \pm 3.90$ & $17.26 \pm 3.29 * * *$ \\
\hline
\end{tabular}

Fig. 1. Pathological examination of the rat cardiac apex. H\&E (upper panels) and Masson's trichrome (lower panels) staining of myocardial tissues at 2, 4, and 6 weeks after surgery. MI: myocardial infarction group. Images were photographed at x200 magnification.

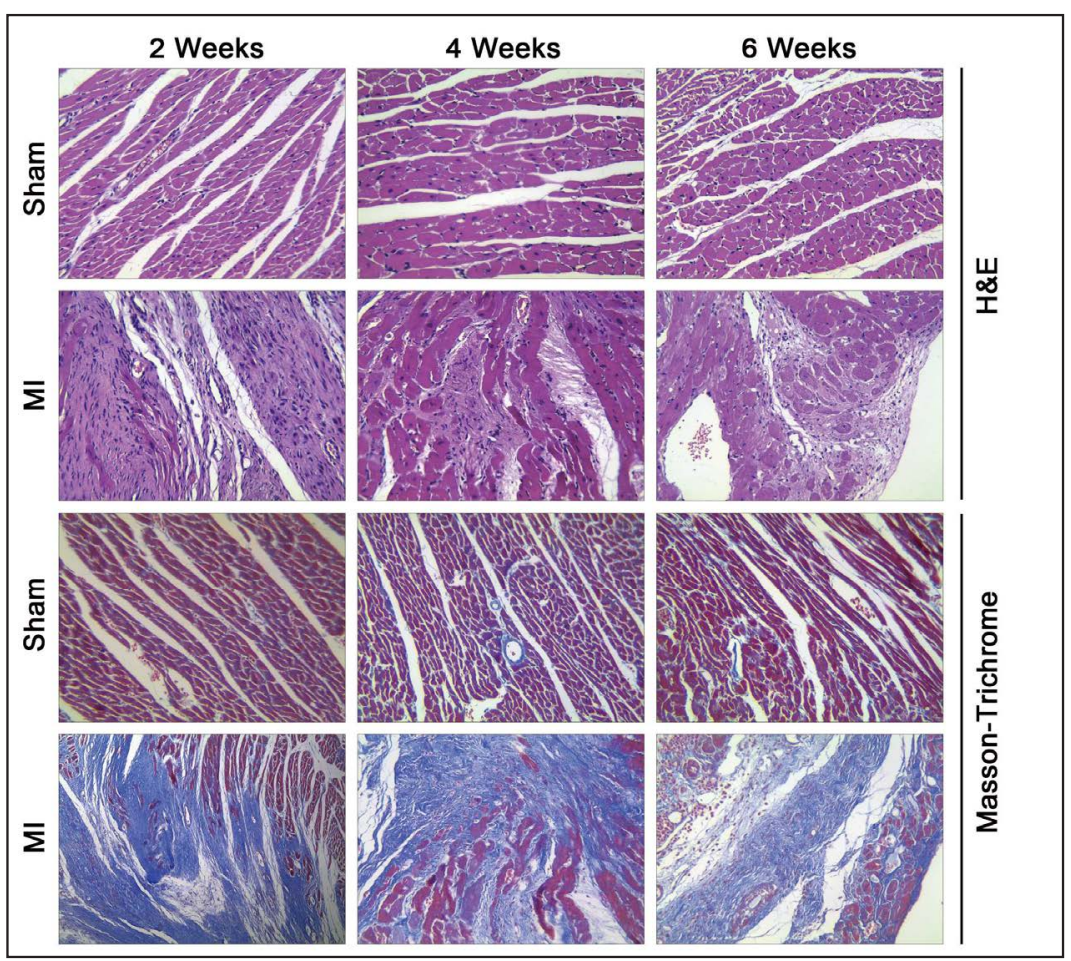

the corresponding SO groups $(\mathrm{P}<0.001)$. However, the degree to which CCK levels were increased in the MI groups compared with the SO groups was greater in the 4-week group than in the 2 -and 6-week groups $(\mathrm{P}<0.01)$. There was no significant difference in BNP levels among the three MI groups.

CCK mRNA and protein expression levels and BNP protein expression levels in the noninfarcted area of the left ventricle

The CCK to GAPDH ratio was not different between the 2-week MI and SO groups; however, CCK expression levels were significantly higher in the 4-and 6-week MI groups than in the corresponding SO groups $(\mathrm{P}<0.001)$. Additionally, CCK expression levels were 


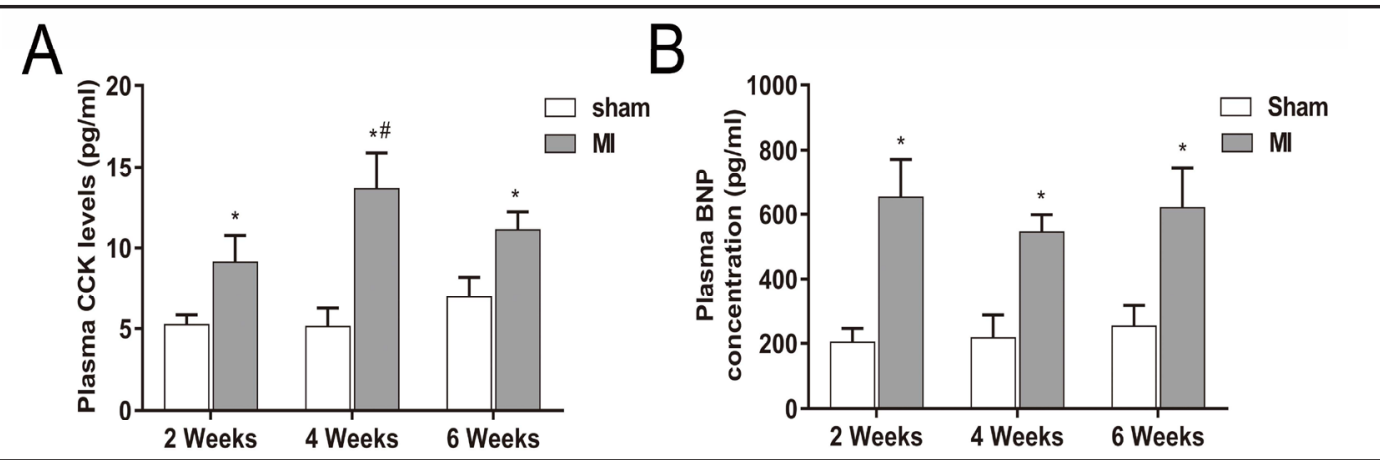

Fig. 2.Changes in plasma CCK (A) and BNP (B) concentrations at different time points after surgery. All values are expressed as the mean \pm SD $(n=5)$. MI: myocardial infarction groups. ${ }^{*} \mathrm{P}<0.001$ versus the timematched sham group, ${ }^{\mathrm{P}}<0.01$ versus the 2 - and 6-week MI groups.

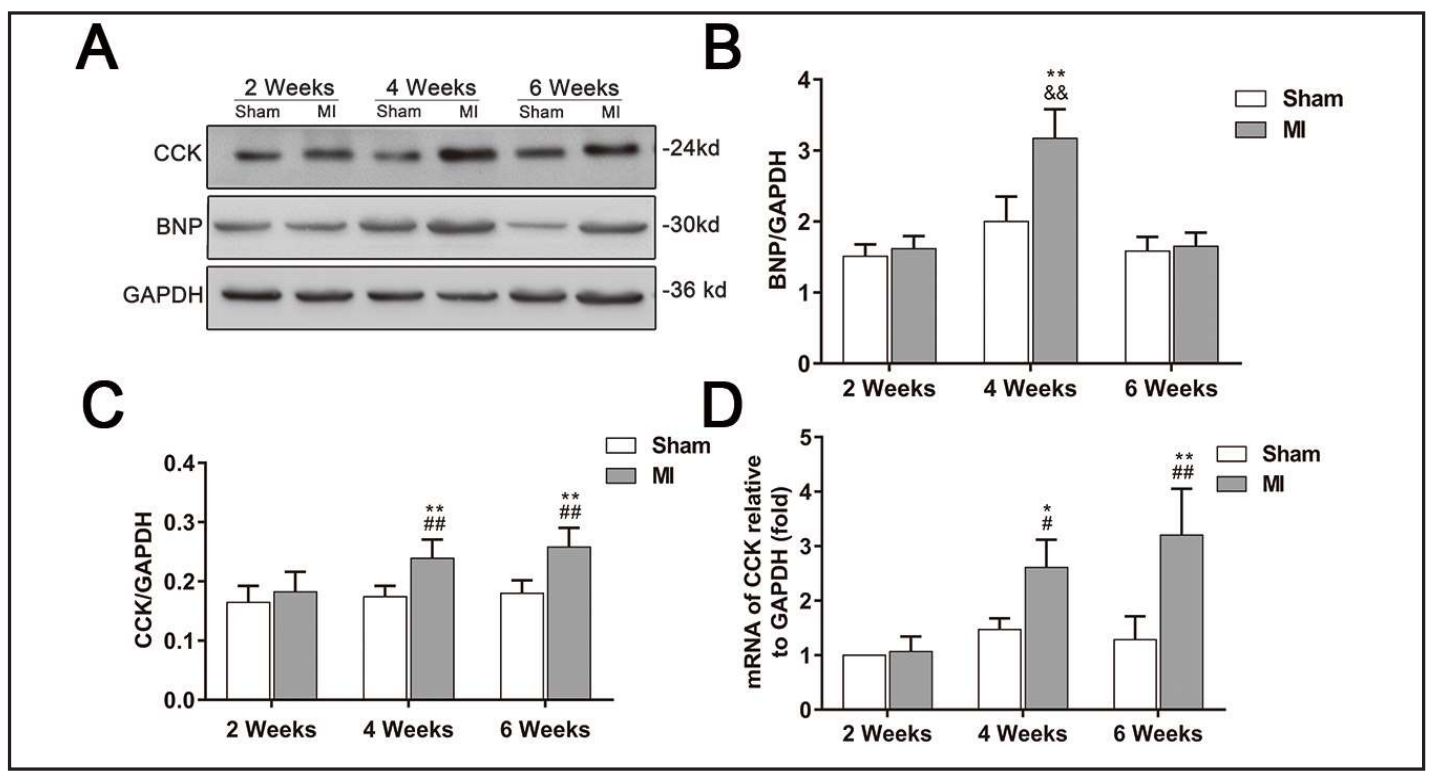

Fig. 3.The noninfarcted area of the left ventricle were collected at the end of treatment and analyzed by RT-PCR and Western blotting. (A) Analysis of BNP, CCK and GAPDH protein expression from the noninfarcted area of the left ventricle samples by Western blot. (B-C) Bar graphs show relative intensity of BNP to GAPDH and CCK to GAPDH respectively. (D) The bars indicate the CCK mRNA levels; GAPDH: glyceraldehyde-3-phosphate dehydrogenase; CCK: cholecystokinin; BNP: brain natriuretic peptide; MI: myocardial infarction groups. The dates were summarized from 3 independent experiments. All values are represented as the mean $\pm \mathrm{SD}(\mathrm{n}=3) .{ }^{*} \mathrm{P}<0.05,{ }^{* *} \mathrm{P}<0.001$, compared with the time-match sham surgery groups; ${ }^{\mathrm{P}}<0.01$,

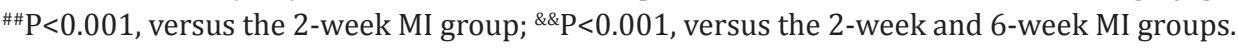

higher in the 4- and 6-week MI groups than in the 2-week MI group. BNP expression levels were significantly increased in 4-week MI rats compared with 4-week SO rats and 2- and 6 -week MI rats $(\mathrm{P}<0.001)$. However, BNP expression levels were not different between the 2-week MI and SO groups, nor were they different between the 6-week MI and SO groups. Quantitative analysis of cardiac CCK mRNA expression levels in the myocardium, which was performed by real-time PCR, showed that CCK expression levels were significantly different between the MI and corresponding SO groups at 4 and 6 weeks after surgery $(\mathrm{P}<0.05$ and $\mathrm{P}<0.001$, respectively); however, CCK mRNA expression levels were not different between the MI and SO groups at 2 weeks after surgery. Moreover, the degree to which CCK mRNA

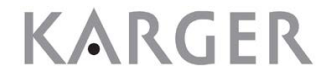


Fig. 4. CCK expression levels were detected by immunohistochemical staining (x400 magnification). MI: myocardial infarction group.

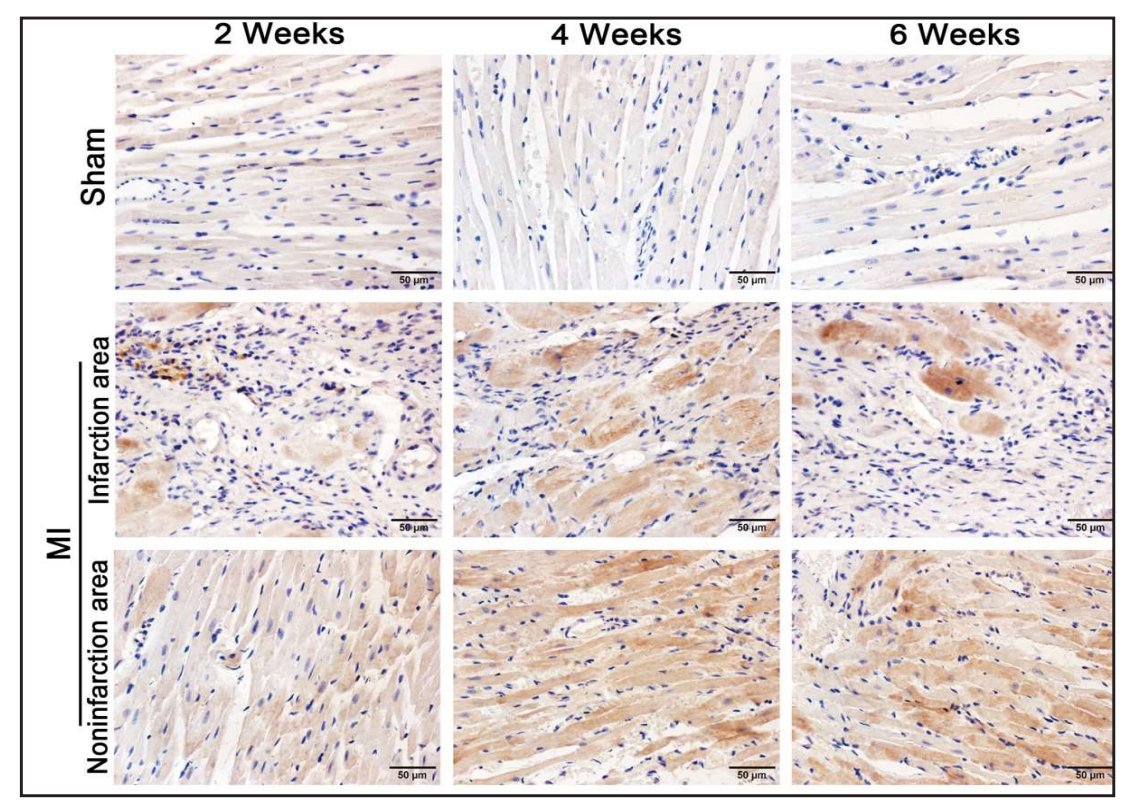

Fig. 5. Correlations between plasma CCK and BNP levels and cardiac functional parameters in model rats. LVEF: left ventricular ejection fraction; LVFS: left ventricular short-axis fractional shortening; LVDs: left ventricular end-systolic diameter; BNP: brain natriuretic peptide. Table 1. Body weights and cardiac functional and structural parameters in the rat models at the end of
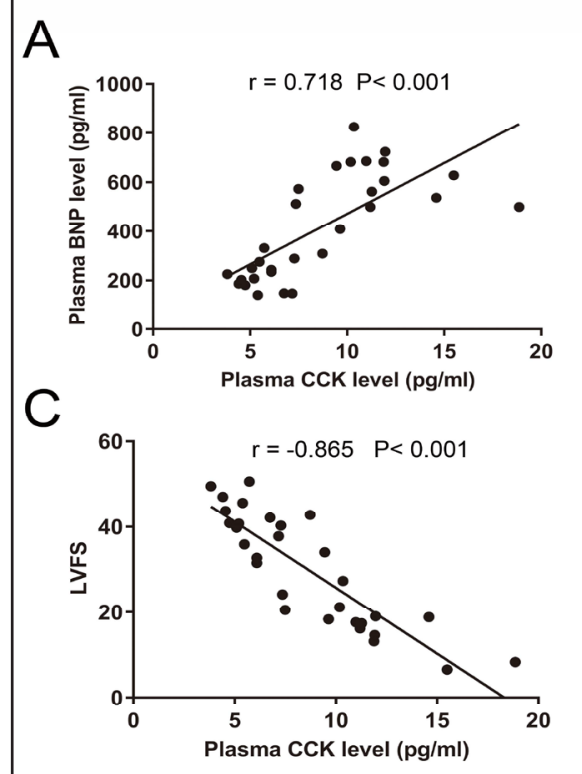

B

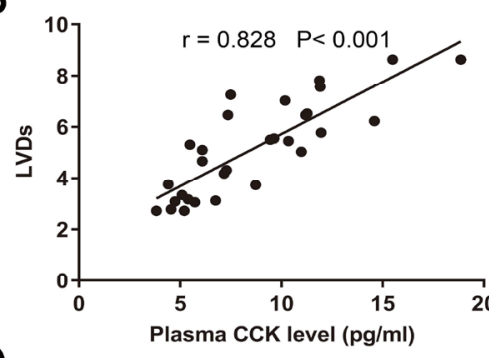

$D$

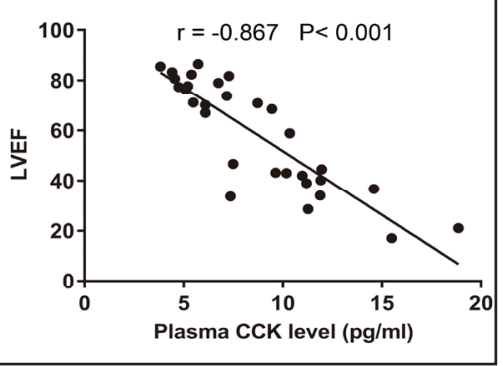
the study. BW: body

weight; HW: heart weight; LA diameter: left atrial diameter; IVSTd: end-diastolic interventricular septal thickness; LVDd: left ventricular end-diastolic diameter; LVDs: left ventricular end-systolic diameter; LVPWTd: left ventricular end-diastolic posterior wall thickness; LVEF: left ventricular ejection fraction; LVFS: left ventricular short-axis fractional shortening; MI: myocardial infarction group. Data are expressed as the mean \pm standard deviation (SD). ${ }^{*} \mathrm{P}<0.05,{ }^{* *} \mathrm{P}<0.01,{ }^{* * *} \mathrm{P}<0.001$, compared with the time-matched SO groups.

levels differed between the MI and SO groups was greater in the 4- and 6-week groups than in the 2-week group (Fig. 3).

\section{Detection of CCK expression by immunohistochemical staining}

CCK was expressed at low levels in the myocardial tissues of the SO groups. Staining for CCK was significantly increased in the infarcted and noninfarcted areas of the left ventricle in the 4- and 6-week MI groups compared with those in the corresponding SO groups (Fig. 4). 


\section{Cellular Physiology Cell Physiol Biochem 2017;43:2479-2488

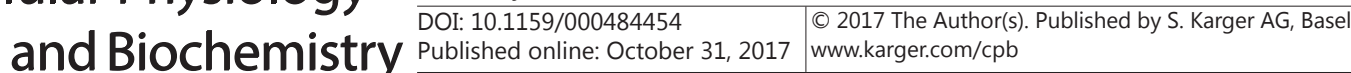 \\ Dong et al.: Expression of Cholecystokinin During Heart Faiure Development}

Associations between plasma CCK levels and heart failure parameters

The correlations between CCK levels and BNP levels and cardiac functional parameters are shown in the indicated figure (Fig. 5). Plasma CCK levels were positively correlated with BNP levels $(\mathrm{P}<0.001)$ and LVDs $(\mathrm{P}<0.001)$ and significantly negatively correlated with the $\mathrm{EF}(\mathrm{P}<0.001)$ and $\mathrm{SF}(\mathrm{P}<0.001)$ in the SO and MI groups.

\section{Discussion}

Increased plasma CCK levels are associated with higher cardiovascular mortality [26]; however, the changes in CCK expression that occur during the development of postinfarction heart failure are unknown. The present study showed for the first time that the development of postinfarction heart failure was associated with significant changes in both CCK mRNA expression and CCK protein expression. Furthermore, plasma CCK levels were positively correlated with BNP concentrations and LVDs and significantly negatively correlated with the EF and SF in rats after surgery.

As a classical brain-gut peptide, CCK and its receptors can be found in a variety of tissues and organs. In addition to having the ability to exert regulatory effects on digestion and the central nervous system [19], CCK also appears to have several effects on the cardiovascular system, although the mechanisms underlying these effects remain unknown [29]. Cholecystokinin octapeptide (CCK-8) has been considered a minor biologically active fragment and has been shown to significantly improve cardiac function and hypotension in rats with lipopolysaccharide-induced endotoxic shock [30], most likely through the CCKAR/BR, which is expressed on cardiac myocytes [25]. A recent clinical study investigated the prognostic value of plasma CCK levels in patients with symptoms of cardiac disease and suggested that plasma CCK levels are an independent biomarker of cardiovascular mortality in elderly female patients [26].

In the present study, we assessed CCK expression levels at multiple time points during the development of heart failure induced by LAD ligation in rats. Ischemic heart disease is the most prevalent cause of HF in humans, and the model enables precise observations of the timing of endocrine and heart function changes that are associated with heart failure progression. We observed higher CCK levels in the plasma in all the MI groups compared with the corresponding SO groups. We also observed that increases in plasma CCK levels were associated with corresponding changes in heart functional parameters and BNP levels. Plasma CCK levels are elevated in rats with heart failure, suggesting that CCK may be an indicator of myocardial injury. Unfortunately, measurements of the levels of the different molecular forms of CCK in plasma were not possible in the present study. The mechanism by which plasma CCK concentrations increase in rats with heart failure is unclear. Our results support the hypothesis that the rise in plasma CCK levels that occurs in heart failure may be due to the release of CCK from myocardial tissue; however, we cannot exclude the possibility that other factors involved in regulating CCK release may participate in the development of MI. Additionally, whether circulating CCK levels reflect myocardial CCK levels remains to be determined. Plasma BNP concentrations in the MI groups were significantly higher than those in the SO groups; however, BNP protein expression levels in the myocardium were not significantly different between the 2- and 6-week groups. These divergent results may indicate that BNP located in ventricular myocardial granules is rapidly secreted or degraded.

In this study, we demonstrated that CCK protein expression levels were significantly increased in the noninfarcted area of the left ventricle in the MI groups compared with the matched SO groups at 4 and 6 weeks after MI. We also measured CCK mRNA expression levels by qRT-PCR to determine whether the changes in CCK mRNA expression levels that occurred after MI were consistent with those that occurred in CCK protein expression levels in model animals. We found that CCK mRNA expression levels were significantly increased in the 4- and 6-week MI groups compared with the time-matched SO groups. Furthermore, immunohistochemical staining of the rat myocardium showed that CCK expression levels in the left ventricle were significantly increased in the 4- and 6-week MI groups compared with 


\section{Cellular Physiology Cell Physiol Biochem 2017;43:2479-2488

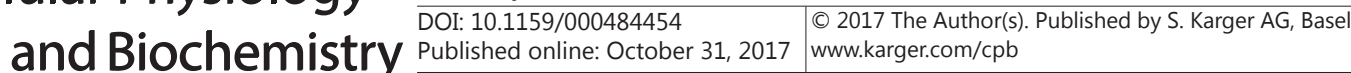 \\ Dong et al.: Expression of Cholecystokinin During Heart Faiure Development}

the corresponding SO groups. A previous report demonstrated that cardiac CCK expression is significantly regulated by isoprenaline [21]; thus, the significant increases in CCK mRNA and protein expression in the noninfarcted area of the left ventricle that were noted herein may reflect adaptions that increase sympathetic activity during heart failure progression. Upregulated CCK may stimulate aldosterone secretion [31, 32]. Therefore, upregulated CCK may have autocrine or paracrine effects in the pathogenesis of heart failure.

This study did not describe infract sizes, which may affect CCK expression levels. However, our combined immunohistochemical (IHC) staining, western blotting, real-time PCR and ELISA data enabled us to perform an accurate assessment of the changes in CCK expression that are associated with the development of postinfarction heart failure. The results obtained from the heart failure rat models provide the basic experimental data and evidence for further studies in other species and clinical patients. Future studies should elucidate the exact mechanisms and physiological targets of the CCK system in the heart.

\section{Conclusion}

In conclusion, this study showed that the development of postinfarction heart failure is associated with changes in CCK expression and that upregulated CCK levels, which were correlated with heart functional parameters and BNP levels, may play a role in heart failure progression after infarction. Thus, CCK may serve as a promising target for the evaluation and treatment of heart failure.

\section{Acknowledgements}

The authors thank Jiemei Yang for kindly assisting with the echocardiographic studies.

\section{Disclosure Statement}

The authors have no financial conflicts of interest.

\section{References}

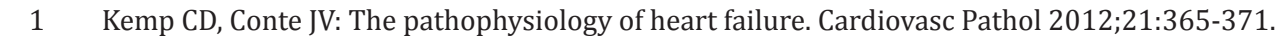

-2 Francis J, Weiss R, Wei S, Johnson A, Felder R: Progression of heart failure after myocardial infarction in the rat. Am J Physiol Regul Integr Comp Physiol 2001;281:R1734-R1745.

3 Khatib MN, Shankar A, Kirubakaran R, Agho K, Simkhada P, Gaidhane S, Saxena D, B U, Gode D, Gaidhane A, Zahiruddin SQ: Effect of ghrelin on mortality and cardiovascular outcomes in experimental rat and mice models of heart failure: a systematic review and meta-analysis. PLoS One 2015;10:e0126697.

4 Nagaya N, Uematsu M, Kojima M, Date Y, Nakazato M, Okumura H, Hosoda H, Shimizu W, Yamagishi M, Oya H, Koh H, Yutani C, Kangawa K: Elevated Circulating Level of Ghrelin in Cachexia Associated With Chronic Heart Failure: Relationships Between Ghrelin and Anabolic/Catabolic Factors. Circulation 2001;104:20342038.

5 Lenk K, Palus S, Schur R, Datta R, Dong J, Culler MD, Anker S, Springer J, Schuler G, Adams V: Effect of ghrelin and its analogues, BIM-28131 and BIM-28125, on the expression of myostatin in a rat heart failure model. J Cachexia Sarcopenia Muscle 2013;4:63-69.

6 Luo LJ, Liu YP, Yuan X, Zhang GP, Hou N, Wu XQ, Luo JD, Zhang GS: Leptin Attenuates the Contractile Function of Adult Rat Cardiomyocytes Involved in Oxidative Stress and Autophagy. Acta Cardiol Sin 2016;32:723-730.

7 von Haehling S, Doehner W, Anker SD: Nutrition, metabolism, and the complex pathophysiology of cachexia in chronic heart failure. Cardiovasc Res 2007;73:298-309.

-8 Cong B, Li SJ, Ling YL, Yao YX, Gu ZY, Wang JX, You HY: Expression and cell-specific localization of cholecystokinin receptors in rat lung. World J Gastroenterol 2003;9:1273-1277. 


\section{Cellular Physiology Cell Physiol Biochem 2017;43:2479-2488

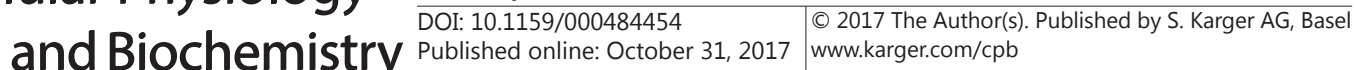

-9 LAY JM, GILLESPIE PJ, SAMUELSON LC: Murine Prenatal expression of cholecystokinin in Neural Crest, Enteric Neurons, and Enteroendocrine Cells. Dev Dyn 1999;216:190-200.

10 Rehfeld JF: Cholecystokinin-From Local Gut Hormone to Ubiquitous Messenger. Front Endocrinol (Lausanne) 2017;8:47.

11 Rehfeld JF, Friis-Hansen L, Goetze JP, Hansen TV: The biology of cholecystokinin and gastrin peptides. Curr Top Med Chem 2007; 7:1154-1165.

12 Chandra R, Liddle RA: Cholecystokinin. Curr Opin Endocrinol Diabetes Obes 2007;14:63-67.

13 Liddle RA: cholecystokinin: its role in health and disease. Curr Opin Endocrinol Diabetes Obes 2003;10:5054.

14 Tian F, Ling Y, Chen Y, Wang Z: Effects of CCK-8 and Cystathionine gamma-Lyase/Hydrogen Sulfide System on Acute Lung Injury in Rats. Inflammation 2017;40:174-183.

15 Aunapuu M, Roosaar P, Jarveots T, Kurrikoff K, Koks S, Vasar E, Arend A: Altered renal morphology in transgenic mice with cholecystokinin overexpression. Transgenic Res 2008;17:1079-1089.

16 Zhang Y, Zhu J, Guo L, Zou Y, Wang F, Shao H, Li J, Deng X: Cholecystokinin protects mouse liver against ischemia and reperfusion injury. Int Immunopharmacol 2017;48:180-186.

17 Noble F, Wank SA, Crawley JN, Bradwejn J, Seroogy KB, Hamon M, Roques BP: International Union of Pharmacology. XXI. Structure, distribution, and functions of cholecystokinin receptors. Pharmacol Rev 1999;51:745-781.

18 Weng Y, Sun S, Song F, Phil Chung S, Park J, Harry Weil M, Tang W: Cholecystokinin octapeptide induces hypothermia and improves outcomes in a rat model of cardiopulmonary resuscitation. Crit Care Med 2011;39:2407-2412.

-19 Mhalhal TR, Washington MC, Newman K, Heath JC, Sayegh AI: Infusion of exogenous cholecystokinin-8, gastrin releasing peptide-29 and their combination reduce body weight in diet-induced obese male rats. Appetite 2017;109:172-181.

20 Zhang D, Li H, Geng J, Li Y, Li S, Ma C, Cong B, Zhang X: The therapeutic effects of cholecystokinin octapeptide on rat liver and kidney microcirculation disorder in endotoxic shock. Immunopharmacol Immunotoxicol 2017;39:2-10.

-21 Goetze JP, Johnsen AH, Kistorp C, Gustafsson F, Johnbeck CB, Rehfeld JF: Cardiomyocyte expression and cellspecific processing of procholecystokinin. J Biol Chem 2015;290:6837-6843.

22 Lovick TA: CCK as a modulator of cardiovascular function. J Chem Neuroanat 2009;38:176-184.

23 Mikulášková B, Maletínská L, Zicha J, Kuneš J: The role of food intake regulating peptides in cardiovascular regulation. Mol Cell Endocrinol 2016;436:78-92.

24 Ruiz-Gayo M, Gonzalez MC, Fernandez-Alfonso S: Vasodilatory effects of cholecystokinin: new role for an old peptide? Regul Pept 2006;137:179-184.

25 Zhao XY, Ling YL, Li YG, Meng AH, Xing HY: Cholecystokinin octapeptide improves cardiac function by activating cholecystokinin octapeptide receptor in endotoxic shock rats. World J Gastroenterol 2005;11:3405-3410.

-26 Goetze JP, Rehfeld JF, Alehagen U: Cholecystokinin in plasma predicts cardiovascular mortality in elderly females. Int J Cardiol 2016;209:37-41.

-27 Gray GA, Sherry L: Investigation of the endothelin system in experimental heart failure. Methods Mol Biol 2002;206:217-227.

28 Schiller NB, Shah PM, Crawford M, DeMaria A, Devereux R, Feigenbaum H, Gutgesell H, Reichek N, Sahn D, Schnittger I, Silverman NH, Tajik AJ: Recommendations for Quantitation of the Left Ventricle by TwoDimensional Echocardiography. J Am Soc Echocardiogr 1989;2:358-367.

29 Wisniewska RJ, Wisniewski K: Cholecystokinin (CCK) and C-terminal fragments of CCK: effects of CCK-33, CCK-8 and CCK-4 in the cardiovascular system of diabetic rats. Gen Pharmacol 1996;27:399-405.

30 Saia RS, Bertozi G, Mestriner FL, Antunes-Rodrigues J, Queiroz Cunha F, Carnio EC: Cardiovascular and inflammatory response to cholecystokinin during endotoxemic shock. Shock 2013;39:104-113.

-31 Nussdorfer GG, Spinazzi R, Mazzocchi G: Cholecystokinin and Adrenal - Cortex Secretion. Vitam Horm 2005;71:433-453.

32 Malendowicz LK, Nowak M, Gottardo L, tortorella C, Majchrzak M, Nussdorfer GG: Cholecystokinin Stimulates Aldosterone Secretion from Dispersed Rat Zona Glomerulosa Cells, Acting Through Cholecystokinin Receptors 1 and 2 Coupled with the Adenylate Cyclase Dependent Cascade. Endocrinology $2001 ; 142: 4251-4255$. 